Drucken. In diesem Fall ist im Verbrennungsgas eine Überkonzentration an Radikalen gegenüber den Gleichgewichtswerten vorhanden.

Da im Falle der stark leuchtenden Acetylenflamme die gemessene Temperatur genügend genau mit der berechneten übereinstimmt, kann gefolgert werden, daß offenbar eine Übertragung von Reaktionswärme auf die Rußteilchen im Elementarakt der Rußbildung nicht stattfindet, denn im anderen Falle wäre eine „Chemielumineszenzstrahlung“ des Rußes zu erwarten, die auch im Falle hoher Rußdichte eine Übertemperatur des Rußes ohne Mitwirkung von Radikalen ergeben müßte.

Die Existenz einer hohen Übertemperatur der Rußteilchen im Leuchtstrahl der Benzolflamme zeigt andererseits, daß der Energietransport durch Radikaldiffusion in Flammen neben der normalen Wärmeleitung von Bedeutung werden kann. Ein ähnlicher
Vorgang ist schon bei der Wärmeleitung dissoziierender Gase bekannt, wo die Wärmeleitung infolge Diffusion von Dissoziationsprodukten auf das Zehnfache des normalen Wertes gesteigert werden kann ${ }^{14}$. In unserem Falle stellt sich ein stationäres Gleichgewicht ein. Der normale Wärmeübergang zwischen Rußteilchen und Gas strebt einen Temperaturausgleich an, die Radikalrekómbination an den Rußteilchen bewirkt eine Erhitzung der Teilchen. Eine merkliche Übertemperatur wird immer dann resultieren, wenn die Rekombination und damit die Übertragung der Rekombinationswärme neben dem normalen Wärmeübergang eine nennenswerte Rolle spielt.

14 Vgl. A. E u cke n, Lehrbuch der chemischen Physik, II, 1, S. 325, 1943. Ähnliche Verhältnisse liegen in den Plasmen der Hochdruckentladungen vor, wo ein gewisser Anteil der Wärmeleitung durch Diffusion von Ionen zustande kommt.

\title{
Entmischungskurven von Polystyrollösungen
}

\author{
Von Ernst Jenckel und Günther Keller \\ Aus dem Institut für theoretische Hüttenkunde und physikalische Chemie \\ der Techn. Hochschule Aachen \\ (Z. Naturforschg. 5 a, 317-321 [1550]; eingegangen am 9. Dezember 1949)
}

\begin{abstract}
Hochmolekulare Lösungen entmischen sich in kennzeichnender Weise derart, daß die kritische Zusammensetzung weit zur Seite des reinen Lösungsmittels verschoben ist.

Es wurden die Löslichkeitskurve und insbesondere der kritische Punkt in den Lösungen des Polystyrols mit einer Reihe beschränkt lösender Lösungsmittel bestimmt. In homologen Reihen der Lösungsmittel scheint die kritische Temperatur zunächst zu sinken, um nach Durchschreiten eines Minimums wieder anzusteigen, wie sich besonders gut an den Alkoholen zeigen ließ. Die kritische Zusammensetzung läßt noch keinen Zusammenhang mit der chemischen Konstitution erkennen. Mit der Kettenlänge des Polystyrols steigt die kritische Temperatur nur sehr wenig an, die kritische Zusammensetzung ändert sich nicht merklich.
\end{abstract}

$D^{i}$ ie hochmolekularen Lösungen sind sowohl vom wissenschaftlichen wie vom technischen Standpunkt aus von Interesse, wobei hier nur an viskosimetrische und osmotische Untersuchungen und an solche über Anstriche und Weichmacher erinnert sei. Manche Lösungsmittel (LM) lösen bei tieferer Temperatur nur beschränkt und vollkommen erst nach Überschreiten einer kritischen Temperatur. Sie verhalten sich also wie z. B. Phenol und Wasser. Solche beschränkt mischbaren LM sind nicht oft untersucht worden. Im folgenden werden im Anschluß an eine frühere Arbeit ${ }^{1}$ Löslichkeitsbestimmungen des Polystyrols in einer Reihe solcher LM mitgeteilt. — Über

1 E. J e n cke l, Z. Naturforschg. 3a, 290 [1948]. die thermodynamische Berechnung des kritischen Punktes soll später berichtet werden.

\section{A. Herstellung des Styrolpolymerisates}

Das monomere Styrol wurde destilliert und die Fraktion von $149^{\circ} \mathrm{C}$ zur Polymerisation verwandt. $\mathrm{Zu}$ dem Zweck wurden etwa $15 \mathrm{~g}$ im zugeschmolzenen Rohr ohne Katalysator 24 Stdn. auf $200^{\circ}$ erhitzt. Das entstandene Polymerisat wurde in $500 \mathrm{~cm}^{3}$ Toluol gelöst und daraus mit Methanol als zähflüssige, sich unten absetzende Schicht ausgefällt, die nach dem Abtrennen und Waschen mit Methanol fest wurde. Dann wurde das Polystyrol in heiBem $n$-Hexanol-(3) gelöst, aus dem es beim Erkalten in weißen Flocken ausfiel, die sich gut abnutschen ließen. Der Niederschlag wurde ncchmals längere Zeit mit Methanol gekocht, um das anhaftende Hexanol zu entfernen. dann wieder abgenutscht, mehrmals mit Methanol nach- 
gewaschen und schließlich im Vakuum bei $60^{\circ}$ getrocknet. Man erhält so ein feines lockeres weißes Pulver. Zwei auf diese Art getrennt hergestellte Präparate zeigten bei ein und demselben Lösungsmittel keinen Unterschied in der Entmischungstemperatur.

Das Molgewicht wurde viskosimetrisch nach Staudinger ermittelt.

$$
\mathrm{M}=\frac{1}{K_{\mathrm{m}}} \eta_{\mathrm{sp}} \frac{M^{\prime}}{c}
$$

[ $M$ und $M^{\prime}=$ Molgewicht des Polymerisats bzw. des Mcnomeren, $\eta_{\mathrm{sp}}=\left(\eta_{\mathrm{L}}-\eta_{0}\right) / \eta_{0}=$ spezifische Viskosität, $c=$ Konzentration in $\left.\mathrm{g} / l, K_{\mathrm{m}}=0,43 \cdot 10^{-4}\right]^{2}$. In der Lösung von Toluol in einer Konzentration von etwa $2 \mathrm{~g} / \mathrm{l}$ ergab sich $M=62600$ und dementsprechend ein Polymerisationsgrad $P=602$.

B. Übersicht über die Löslichkeit verschiedener Stoffe für Polystyrol

Schon die Faustregel, daß hochmolekulare Stoffe von Lösungsmitteln entweder unbeschränkt oder gar nicht gelöst werden, läßt erkennen, daß es verhältnismäßig wenige Lösungsmittel geben wird, die beschränkt lösen, also im Temperaturgebiet zwischen -40 und $+200^{\circ}$ einen kritischen Punkt haben, oberhalb dessen vollständige gegenseitige Löslichkeit und unterhalb dessen Trennung in zwei flüssige Fhasen beobachtet wird. (Oberhalb von $+200^{\circ}$ beginnen die langen Ketten des Polystyrols merklich zu zerbrechen, unterhalb von $-40^{\circ}$ wird die Geschwindigkeit der Entmischung langsam ${ }^{3}$.)

Orientierende Versuche an einem sehr hochmolekularen technischen Polystyrol zeigten ${ }^{4}$, daß dieses praktisch unlöslich ist in Paraffinen (Petrolbenzin, Paraffinöl) und in hydroaromatischen Kohlenwasserstoffen (Dekalin).

Durch Einführen einer geeigneten funktionellen Gruppe kann man mehr oder weniger gute LM erhalten. Dabei zeigte sich wiederholt, daß neben der funktionellen Gruppe auch die Länge der aliphatischen Kette des LM wichtig ist. Mit der gleichen funktionellen Gruppe geben mittlere Aliphate meist die besseren Löslichkeiten als die niederen und die höheren; z. B. bewirkt die Estergruppe gute Löslichkeit bei mittleren Estern (Propyl-, Butyl-, Amyl-

2 G. V. Schultz u. E. Husemann, Z. phys. Chem. Abt. B 34, 187 [1936].

3 Die Lösungen in Äthylacetat sind bei der Entmischungstemperatur von $-31^{\circ}$ und weniger bei einer Konzentration von etwa 8 Grundmolproz. schon fast steif. Es beginnt dann die Trübung an einem Punkte und schreitet von dort nach allen Seiten mit mäßiger Geschwindigkeit fort, ähnlich einem Kristallisationsvorgang. Es hat also nicht viel Zweck, auf noch tiefere Temperaturen herunterzugehen. acetat; Äthyl-, Amyl-butyrat; Amylacrylat; Vinylisobutyrat). Das gilt auch für die Ester zweibasischer Säuren (Adipinsäure-dimethylester). Beschränkte Löslichkeit ergeben niedere Ester (Methylacetat) und höhere Ester (Stearinsäure-butylester). Die Alkoholgruppe bewirkt stets nur beschränkte Löslichkeit, am besten ist sie jedoch bei den Alkoholen mit 8 oder 10 Kohlenstoffatomen ${ }^{5}$. Das gleiche gilt vielleicht für die Doppelbindung (Octylen). Die COOH-Gruppe führt in den niederen Gliedern (Essigsäure, Propionsäure, Brenztraubensäure, Methacrylsäure) nicht zum Lösevermögen, erst eine höhere Säure löst beschränkt (Capronsäure), während die noch höhere Elaidinsäure wieder nicht löst. Dimethylketon löst nicht, Äthylmethylketon löst besser. Weitere Beispiele für beschränkte Löslichkeit sind aus den weiter unten angeführten Messungen ersichtlich.

Die Einführung des Benzolringes führt in jedem Fall zu guter Löslichkeit, und ebenso wirken die heterocyclischen Verbindungen (Furan, Tiophen, Pyridin, Dioxan) und ihre Hydrierungsprodukte (Tetrahydrofuran, Piperidin). Auch alle Chlorderivate lösen gut, mit Ausnahme des Acetylenchlorids, das nicht löst. - Acetonitril und Paraldehyd lösen nicht.

Bei diesen Versuchen fielen die oft beträchtlichen Unterschiede der Auflösegeschwindigkeit und des Lösungsvorganges auf ${ }^{6}$. Die eine Gruppe der LM löst, wie bei einem normalen Lösevorgang, ständig die Oberfläche des Bodenkörpers weg, bis alles aufgelöst ist. Die andere Gruppe durchdringt den Bodenkörper erst völlig, quillt ihn dann auf, so daß er durchscheinend wird und vom LM optisch kaum noch zu unterscheiden ist, und löst ihn dann langsam auf. Eine gesetzmäßige Einteilung der LM konnte nicht durchgeführt werden, jedoch liegen die der ersten Art mehr bei den guten (Benzol, Toluol), die der zweiten Art mehr bei den schlechten Lösungsmitteln (Ester).

C. Bestimmung der Entmischungskurven

An Lösungsmitteln wurden in der Hauptsache Ester Alkohole und Säuren benutzt. Sie wurden möglichst fraktioniert, was aber wegen der häufig nur in geringen Mengen zur Verfügung stehenden Substanzen nicht immer möglich war. Geringe Verunreinigungen der Lösungs-

4 vgl. auch: H. G n a m m, Lösungs- und Weichmachungsmittel, Stuttgart 1941, und H. S t a u d i n g e r u. W. H e u e r, Z. phys. Chem., Abt. A 171, 129 [1934].

5 Auch bei den Paraffinen scheinen die mittleren Glieder noch am besten zu lösen (Petroleum, kritische Temp. bei $180^{\circ}$ ).

6 vgl. auch H. Sta u dinger, Organische Kolloidchemie, Braunschweig 1941, S. 116. 
mittel werden sich aber um so weniger auswirken, je näher die Entmischungspunkte für das reine Lösungsmittel und für die beigemengte Verunreinigung beieinander liegen. Da Polystyrol ein stark hydrophober Stoff ist, wurden fast alle Lösungsmittel noch besonders mit Calciumchlorid oder Kaliumcarbonat getrocknet. Gelegentlich wurde die Reinheit durch eine Schmelzpunktsbestimmung kontrolliert.

Die Mischungen wurden jeweils durch Abwägen von Polystyrol und etwa 0,5 g des Lösungsmittels im Einschmelzröhrchen hergestellt. Das Mischungsverhältnis wird in Grundmolenbrüchen $x_{2}^{\prime}$ angegeben, um sich so von dem nicht genau zu bestimmenden Molgewicht des Polymerisats unabhängig zu machen. Es gilt also:

$$
x_{2}{ }^{\prime}=\frac{m_{2} / M^{\prime}}{m_{2} / M^{\prime}+m_{1} / M_{1}} ; \quad x_{1}{ }^{\prime}+x_{2}{ }^{\prime}=1,
$$

wobei $m_{2}$ und $m_{1}$ die Menge Polystyrol bzw. Lösungsmittel und $M^{\prime}$ und $M_{1}$ die Molgewichte des monomeren Styrols bzw. des LM bedeuten.

Zur genauen Beobachtung der Entmischungstemperatur diente zwischen 20 und $150^{\circ}$ ein Thermostat mit Paraffinöl als Heizbad. Die Röhrchen wurden auf einen Rahmen gespannt, der durch einen Motor gedreht wurde, so daß dauernd genügende Durchmischung stattfand. Es wurde zunächst soweit aufgeheizt, daß alle Röhrchen klar waren. Dann wurde die Temperatur jeweils um $2 /{ }^{\circ}{ }^{\circ}$ herabgesetzt und dabei der Beginn der Trübung beobachtet. Die Beleuchtung geschah von der Seite her gegen einen dunklen Hintergrund. Es ließ sich so nicht nur die Temperatur genau bestimmen, sondern auch die Reihenfolge des Trübewerdens und damit die Lage der kritischen Zusammensetzung, was sonst für flache Kurven nicht ganz einfach ist.

Bei Temperaturen unterhalb von $20^{\circ}$ bis $30^{\circ}$ wurde ein kleines Dewargefäß mit Methanol benutzt, das man mit Hilfe von fester Kohlensäure stark abkühlen konnte. Die Temperaturkonstanz war ausreichend. Es konnte jeweils jedoch nur ein Röhrchen beobachtet werden mit einer Temperaturgenauigkeit von einem halben Grad. - Bei Temperaturen über $150-180^{\circ}$ wurde Phthalsäure-diäthylester als Heizbad benutzt. Die Temperaturkonstanz war hier nicht so gut, jedoch waren die Differenzen zwischen mehreren Bestimmungen selten größer als ein halbes Grad.

Die Temperatur der Entmischung ist am genauesten in der Umgebung des kritischen Punktes festzustellen. Bei sehr kleinen Konzentrationen, auf dem steilen abfallenden Ast der Kurve, ist eine genaue Festlegung innerhalb einiger Grad naturgemäß nicht möglich. - Bei höherer Konzentration (von etwa $8-10 \%$ an) macht sich bereits einige Grade vor der Entmischung eine Trübung bemerkbar, ohne daß man von einer Phasentrennung, wie sie im Entmischungspunkt auftritt, sprechen könnte. Wahrscheinlich handelt es sich hierbei um kleine Mengen besonders langer Polystyrolketten, die weniger löslich sind und daher bei etwas höherer Temperatur ausfallen. Eine Fraktionierung des Polystyrols bei weiterer Abkühlung unter den kritischen Punkt, also längs der Löslichkeitskurve, wurde in besonderen, hier nicht mitgeteilten Versuchen festgestellt.

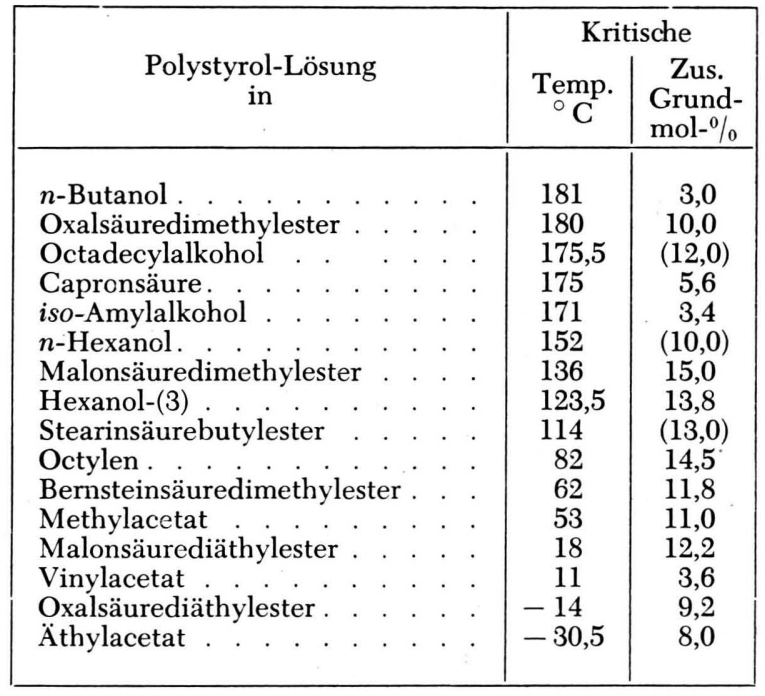

Tab. 1. Kritische Lösungspunkte des Polystyrols in verschienen LM.

\section{Ergebnis se}

\section{Verschiedene Lösungsmittel}

In den Abb. 1 bis 3 sind die beobachteten Entmischungskurven wiedergegeben. Sie zeigen sowohl hinsichtlich der Form als auch der Lage des kritischen Punktes große Unterschiede. Was die Form anlangt, so wurden neben Kurven, die zu einer oder beiden Seiten des kritischen Punktes vollkommen flach verlaufen, z. B. bei den höheren Alkoholen, auch solche gefunden, z. B. Capronsäure, deren Abfall auch zur Seite wachsenden Polystyrolgehalts sehr beträchtlich ist. Während beim Octadecylalkohol in dem untersuchten Konzentrationsbereich bis 53,4 Grundmolprozente die Entmischungstemperatur nur um $3,5^{\circ}$ gefallen ist, beträgt der Abfall bei der Capronsäure bereits bei $20 \% 17^{\circ}$. In Tab. 1 sind die kritischen Daten der untersuchten Lösungen zusammengestellt.

Die kritische Zusammensetzung bewegt sich zwischen fast 1\% (Vinylacetat) und 15\% (Malonsäuredimethylester). Wie ersichtlich, läßt sich bei allen Kurven, mit Ausnahme des Vinylacetats, die kritische Zusammensetzung gut mit Hilfe des geraden Durchmessers festlegen.

Die kritische Temperatur hängt nicht nur von der funktionellen Gruppe ab, sondern ändert sich auch innerhalb einer homologen Reihe manchmal sehr beträchtlich (z. B. bei den Estern). Bei den Alkoholen wurde die kritische Temperatur systematisch verfolgt. Es wurden die Entmischungstemperaturen von Poly- 


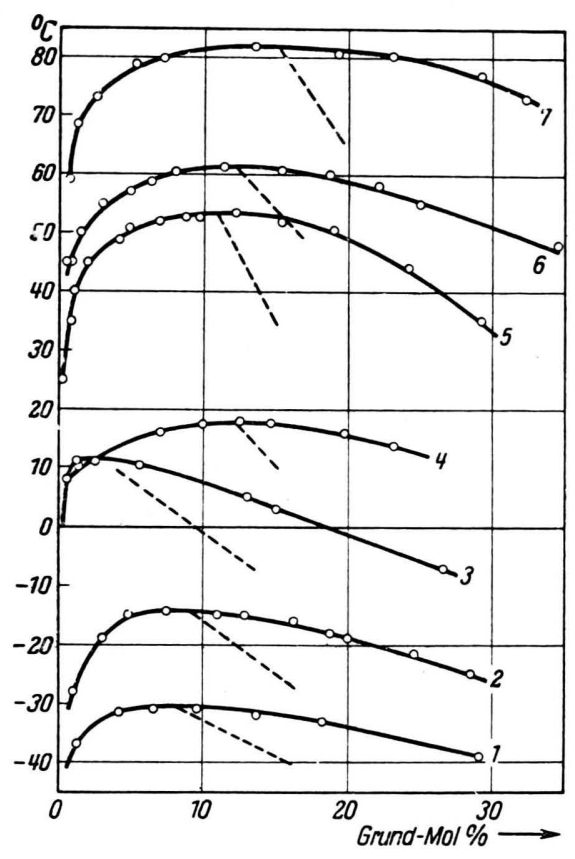

Abb. 1. Entmischungskurven des Polystyrols in verschiedenen LM. 1) Äthylacetat, 2) Oxalsäure - diäthylester, 3) Vinylacetat, 4) Malonsäure - diäthylester, 5) Methylacetat, 6) Bernsteinsäure-dimethylester, 7) Octylen.

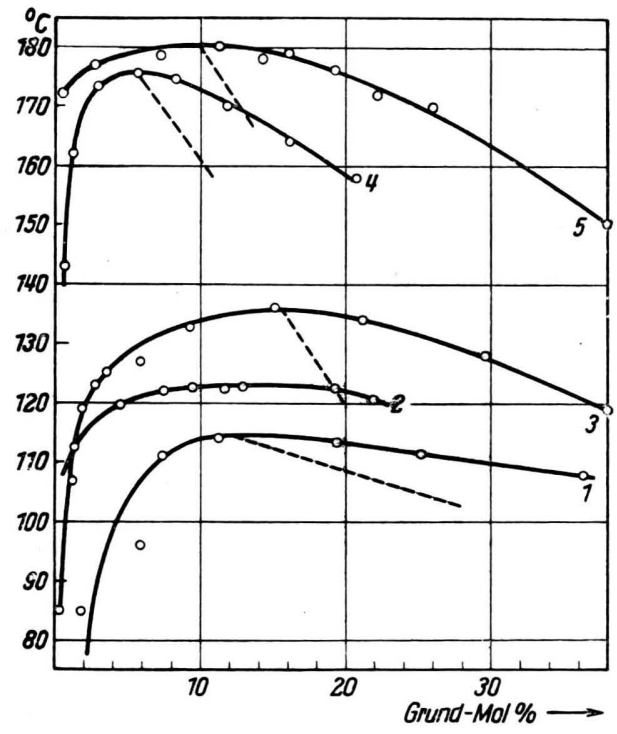

Abb. 2. Entmischungskurven des Polystyrols in verschiedenen LM. 1) Stearinsäure-butylester, 2) Hexanol-(3), 3) Malonsäure-dimethylester, 4) Capronsäure, 5) Oxalsäure-dimethylester.

styrol-Alkohollösungen der Alkohole ${ }^{7}$ mit 4 bis $18 \mathrm{C}$ Atomen untersucht bei etwa der gleichen Konzentration von 8-10 Grundmol-\%, was infolge des flachen Verlaufes der Kurve hinreichend genau ist (Abb. 4).

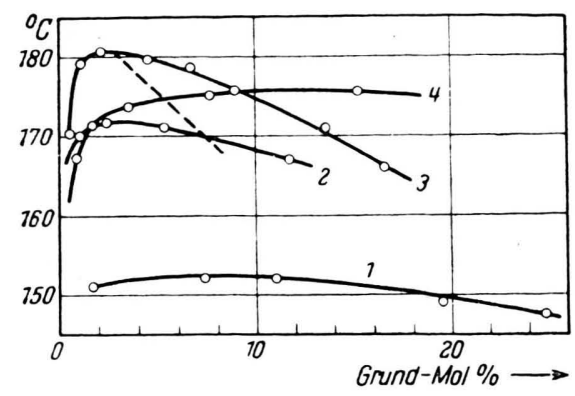

Abb. 3. Entmischungskurven des Polystyrols in verschiedenen LM. 1) n-Hexanol, 2) iso-Amylalkohol, 3) n-Butanol, 4) Octadecylalkohol.

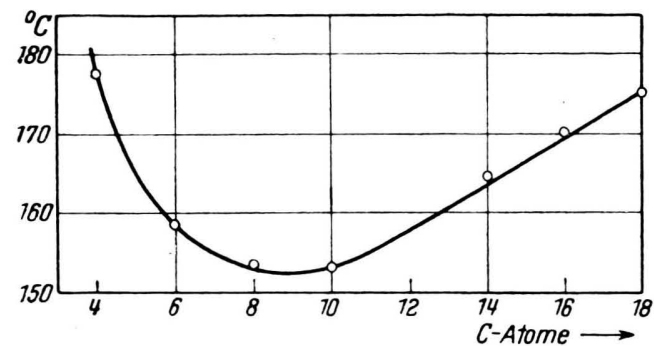

Abb. 4. Entmischungstemperatur des Polystyrols in normalen Alkoholen.

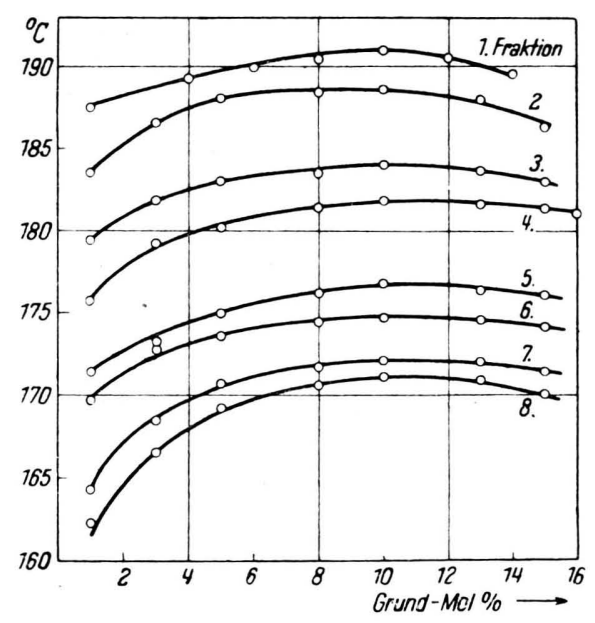

Abb. 5. Entmischungskurven von Fraktionen eines Polystyrols, hergestellt bei $150^{\circ}$, in Octadecylalkohol.

Es standen nur Alkohole mit gerader Kohlenstoffzahl zur Verfügung. Die Alkohole mit ungerader Kohlenstoffzahl könnten, wie von anderen physikalischen Eigenschaften und aus Fällungsversuchen mit

7 Wir danken den $\mathrm{Hy}$ drierwerken $\mathrm{R}$ od le be $\mathrm{n}$ für die Überlassung der Präparate. - Die in dieser Reihe verwandten Präparate $C_{4}$ und $C_{6}$ sind mit den oben benutzten nicht identisch. 


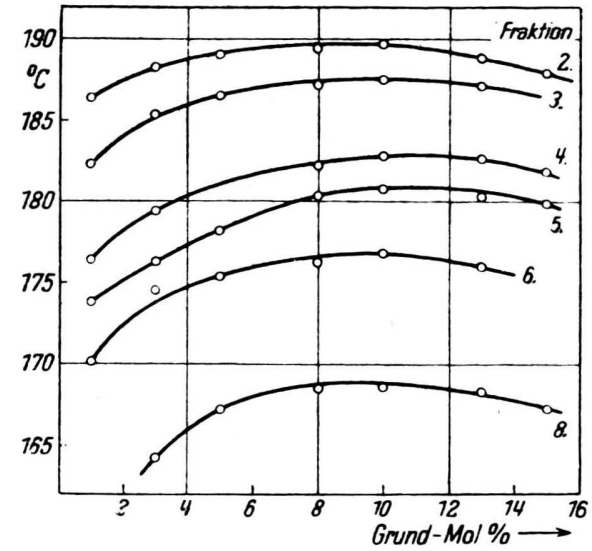

Abb. 6. Entmischungskurven von Fraktionen eines Polystyrols, hergestellt bei $200^{\circ}$, in Octadecylalkohol.

Alkoholen her bekannt ist, außerhalb dieser Reihe liegen. Jedenfalls aber liegt zwischen $\mathrm{C}_{8}$ und $\mathrm{C}_{10}$ ein Minimum; diese Alkohole lösen also am besten.

\section{Verschiedene Polystyrolfraktionen}

In einer anderen Versuchsreihe 8 wurde Styrol bei 150 und $200^{\circ}$ polymerisiert und mit Methanol oder Methyläthylketon fraktioniert ausgefällt. Das Molgewicht wurde viskosimetrisch mit $K_{\mathrm{m}}=0,43 \cdot 10^{-4}$ für das $200^{\circ}$-Polymerisat und $K_{\mathrm{m}}=0,52 \cdot 10^{-4}$ für das $150^{\circ}$-Polymerisat ermittelt und ergab folgende Werte:

Polystyrol $200^{\circ} \quad \begin{array}{llllllll}179 & 205 & 175 & 96 & 83 & 59 & 44 & 30 \cdot 10^{3}\end{array}$ $\begin{array}{llllllllll}\text { Polystyrol } & 150^{\circ} & 155 & 143 & 101 & 60 & 57 & 54 & 37 & 31 \cdot 10^{3}\end{array}$

Die Entmischungskurven dieser Fraktionen wurden in den Lösungsmitteln Octadecylalkohol und Cyclohexanol bestimmt. Sie sind in den Abb. 5 bis 8 wiedergegeben. Übereinstimmend mit dem früheren Befund nimmt die kritische Temperatur nur verhältnismäßig wenig mit steigendem Polymerisationsgrad zu; am stärksten noch bei den kleinen Polymerisationsgraden. Man bekommt eine etwa lineare, aber unterschiedliche Änderung mit $1 / \sqrt{P}$ oder mit $\log P$.

8 durchgeführt von Frl. I r m tra ud Heinen.

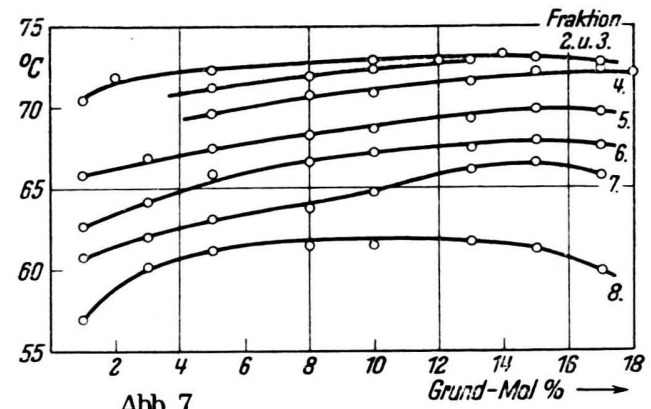

Abb. 7

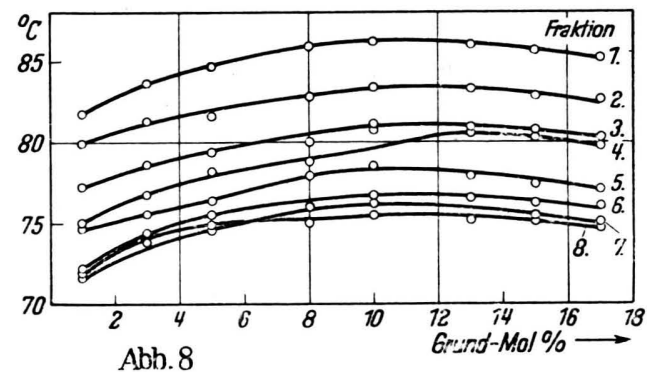

Abb. 7. Entmischungskurven von Fraktionen eines Polystyrols, hergestellt bei $150^{\circ}$, in Cyclohexanol.

Abb. 8. Entmischungskurven von Fraktionen eines Polystyrols, hergestellt bei $200^{\circ}$, in Cyclohexanol.

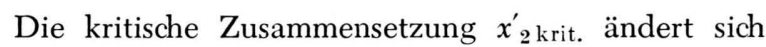
relativ stärker, was aber, wenigstens teilweise, auf die experimentellen Schwierigkeiten zurückzuführen sein mag. In der Lösung des Octadecylalkohols beobachteten wir für das $200^{\circ}$-Polymerisat keine Änderung

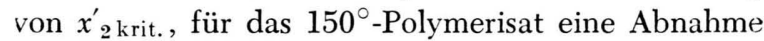
von $11 \%$ auf $10 \%$. In der Cyclohexanol-Lösung wurden am $200^{\circ}$-Polymerisat streuende Werte zwischen 13 und $16,5 \%$, am $150^{\circ}$-Polymerisat ein Anstieg von 10 auf $11,5 \%$ mit steigendem Polymerisationsgrad beobachtet.

9 Warum in der früheren Arbeit eine so viel kleinere kritische Zusammensetzung in der Lösung des Octadecylalkohols beobachtet wurde, konnte noch nicht geklärt werden. 